\title{
Interference Estimated Time of Arrival on a 6-DOF Cable-Driven Haptic Foot Platform
}

\author{
Martin J.-D. Otis, Thien-Ly Nguyen-Dang, Denis Laurendeau and Clément Gosselin
}

\begin{abstract}
A Cable-Driven Locomotion Interface employs two independent cable-driven haptic foot platforms constrained in six degrees of freedom (6-DOF). Its control system and its geometry are designed for performing a wide range of trajectories that could generate cable interferences. This paper presents and analyzes computational methods for determining which cable can be released from an active actuation state while allowing control in a minimal tension state, thereby ensuring that both platforms stay in a controllable workspace. One challaging task is to develop light and fast computational algorithms for hard real time processes included in haptic display applications. Seeing that releasing a cable from an active actuation state might generate discontinuities in tension values in the other cables, this paper proposes collision prediction schemes named Interference Estimated Time of Arrival in order to reduce or completely eliminate such discontinuities.
\end{abstract}

\section{INTRODUCTION}

A Cable-Driven Locomotion Interface (CDLI) has been proposed as a haptic interface to be used in applications such as training in virtual environments [1].

Due to the geometry of cable-driven platforms described in [2] and the trajectories of the feet, cable interferences can occur. As interferences restrict the size of the workspace, various methods were previously devised to analyze them. For instance, Maeda et al. [3] presents the kinematic equations for computing the cable contact point locations, and Merlet [4] develops an algorithm to determine exactly which region of the workspace is interference-free, although cable tension discontinuities were not considered.

A cable can be released from an active actuation state so as to slide on the other cable (called the support cable) during an interference. As a result, platforms are only driven by free cables and support cables. The computation of the tension required on each cable is achieved by an Optimal Tension Distribution (OTD) algorithm that optimizes the wrench (load) to be applied on a platform, as described in [5]. This algorithm can generate tension discontinuities in some cables because the mechanism geometry is modified when a cable is released from its active actuation state.

This paper presents an approach for reducing cable tension discontinuities. The approach is summarized as the action of predicting an interference for adjusting all cable tensions to avoid discontinuities while validating that the platform stays in the Wrench-Closure Workspace (WCW) [6] when a cable is released in order to allow the platform to continue unimpeded on its trajectory. Real cable behaviours, as discribed in

M. J.D. Otis, T.-L. Nguyen-Dang and D. Laurendeau are with the Electrical Engineering Department, Laval University, Quebec, Canada.

C. M. Gosselin is with the Mechanical Engineering Department, Laval University gosselin@gmc.ulaval. ca.
[7], such as cable sagging and cable elongation, are neglected for the same reasons cited in [8].

The first section of this paper presents the cable interference management algorithm that defines the interference and releasing conditions of a cable from the beginning of an interference to its end. The second section presents a method to reduce or eliminate cable tension discontinuities that can occur when a cable is released from an active actuation state. This method computes the intersection of two hyperplanes (in Euclidean space-time) representing the trajectory of each cable participating in a collision, using the known measured derivatives of each platform trajectory at a given time. This allows the computation of the Interference Estimated Time of Arrival (IETA) which in turn can be used to reduce tension discontinuities. Since the system is to be included in a locomotion interface, users must not feel the effect of an interference, which therefore justifies the interference management method for eliminating tension discontinuities.

\section{IMPEDANCE CONTROL Algorithm}

The Cable-Driven Locomotion Interface (CDLI) control algorithm uses a virtual environment (VE) with a physics engine for computing interactions between the platforms and virtual objects as described in Fig. 1: a user applies an action wrench $\mathbf{h}_{a}$ on a platform and on the VE simultaneously, the latter of which responds by a reaction wrench $\mathbf{h}_{r}$ equivalent to the action wrench. The complete control algorithm for hybrid haptic display (impedance and admittance control) was previously presented in [1]. For simplicity purpose, this paper only use Cartesien impedance control of the haptic foot platform.

In Fig. 1, the WCW represents the wrench-closure workspace evaluated for each platform pose and estimated pose using a signal $\mathbf{S}$ that represents the state of all cables (i.e. including free cables, released cables or support cables, labeled 0,1 and 2 respectively in computer simulations as described in Fig.7). OTD generates a set of equilibrium tension values called the setpoint that the cable tension controllers then attempt to follow [9]. The discontinuities that might arise in this process must be reduced or even eliminated because they generate cable vibrations, an undesirable behaviour due to the cable tension controllers overshooting (latency) and oscillating at their natural frequencies, thereby impeding the process of balancing the total wrench and resulting in a deviation of the desired trajectory and in a decrease of haptic display accuracy. Cable tension control is always used whenever some cables are in interference. 
The Cartesian pose $\mathbf{P}_{p f}$ of each platform can be evaluated with the Direct Kinematic Problem (DKP) using the length of the cables $\boldsymbol{\rho}_{m}$ [10]. Finally the IETA box provides an estimated time $\hat{t}$ as well as an estimated position $\mathbf{P}_{p f i}$ of an interference. Its purpose is to reduce or avoid discontinuities by adjusting OTD constraints.

\section{Cable Interference Management Algorithm}

The following section details the interference determination method, which chooses the cable to be released (from an active actuation state) while attempting to reduce tension discontinuities. More precisely, one must determine i) when and where a cable interference begins or ends, ii) which cable must be released from an active actuation state or become the support to the other interfering cable, and iii) how to reduce or eliminate a tension discontinuity at a given interference point.

\section{A. Determination of the interference between cables}

The collision conditions related to an interference point can adopt different mathematical formulations as described in [4], [11] and [3]. One such formulation is based on the minimal distances $L_{m d}$ between all cables (Fig. 2) which can be calculated as follows: the unit vector $\mathbf{n}_{m d}$ describing the minimal distance between two cables is normal to both cables, and can be easily obtained by computing the cross product of the unit vector $\mathbf{n}_{c 2}$ and $\mathbf{n}_{c 1}$ in the direction of each cable, as shown in (1).

A released cable is divided in two sections, one on each side of an interference point on the support cable. It is possible to determine the coordinates of the latter without ever computing the involved forces simply by observing that the length of such a released cable is minimized at all times (assuming that non-ideal behaviour can be neglected) because the controller maintains the cable in minimal tension computed by the OTD algorithm. Extending this approximation, one can assume that the support cable remains straight when minimal tension is applied, which greatly simplifies the computation of the interference point as it simply corresponds to the absolute minimum of the length function describing the released cable. This approximation could be done if the folding angle of the released cable remains low. Of course, both cables in interference have a folding angle with friction and this subject is presented in [12].

The length of the minimal distance vector is derived from the cable attachment point and a system of continuity equations, described by (2):

$$
\begin{gathered}
\mathbf{n}_{m d}=\mathbf{n}_{c 2} \times \mathbf{n}_{c 1} \\
L_{c 1} \mathbf{n}_{c 1}+L_{m d} \mathbf{n}_{m d}-L_{c 2} \mathbf{n}_{c 2}=\mathbf{P}_{m 2}-\mathbf{P}_{m 1}
\end{gathered}
$$

where $L_{c i}$ is the distance between the cable attachment point $\mathbf{P}_{m i}$ of the $i$ th cable and $\mathbf{P}_{c i}$, the intersection with common perpendicular vector $L_{m d} \mathbf{n}_{m d}$. Note that the system in (2) includes three equations, one per spatial dimension, as well as three unknowns: $L_{c 1}, L_{c 2}$ and $L_{m d}$.
The determination of cable interference must consider some non-ideal properties such as cable sagging, finite cable diameter, mechanical deformation of the structure and uncertainties on the position of the cable attachment points. These non-ideal properties are thus taken into account in the relevant algorithms by an effective cable radius $\epsilon_{e}$ defined by (5). The strategy employed is then to release a cable in anticipation of an interference because it is almost impossible in practice to determine errors such as mechanical deformation with enough precision. Hence, the conditions that must be met during interference detection are listed as follows:

- $L_{m d}$ changes sign twice during two computation steps, meaning that the minimal distance $L_{m d}$ has reached 0 during a computation step (equation (3));

- $L_{c 1}$ and $L_{c 2}$ have values ranging between 0 and the full length of their respective cable, meaning that the interference point is not located in the virtual extension of the cables (equation (4));

- $L_{m d}$ is inside a diameter that encompasses the diameter of a cable as well as the errors in position, i.e. cable sagging, deformation, etc. (equation (5))

$$
\begin{aligned}
L_{m d}^{t-1} L_{m d}^{t} & \leq 0 \\
0<L_{c i} & <\left\|\mathbf{P}_{p f i}-\mathbf{P}_{m i}\right\| \\
\left\|L_{m d}\right\| & \leq \epsilon_{e}
\end{aligned}
$$

where $\mathbf{P}_{p f i}$ is the $i$ th cable attachment point on a platform.

When an interference between two cables is detected, one of the two cables is released at the interference point thereby ceasing to drive the platform actively and slides freely on the other cable, which becomes the support cable (see Fig. 2). It is worth noting that the condition $L_{m d}^{t-1} L_{m d}^{t} \leq 0$ described by (3) must never occur, as it implies that an interference is detected a posteriori, i.e., once the two cables have crossed without any cable being released.

Among other things, some interferences such as the contact between two support cables and two released cables are not allowed by the algorithm and thus belong to the Unstable Interference Domain (UID), a set of pathological cases that must be considered at all times during an interference point determination calculation.

\section{B. Selection of the cable to be released}

An interference between two free cables can occur at any time in the course of a trajectory. This interference must be accounted for by choosing which cable needs to be released. The approach that is proposed for implementing the selection process is summarized in Fig. 3, where each step of the algorithm is numbered between 1 to 5 . The steps can be divided as follows: steps 1 and 2 focus on the analysis of the workspace; steps 3 and 5 check the total number of released cables for each platform; and step 4, compares the tension in these both cables.

The integrity of the workspace must be verified first by checking whether or not releasing a given cable from an 


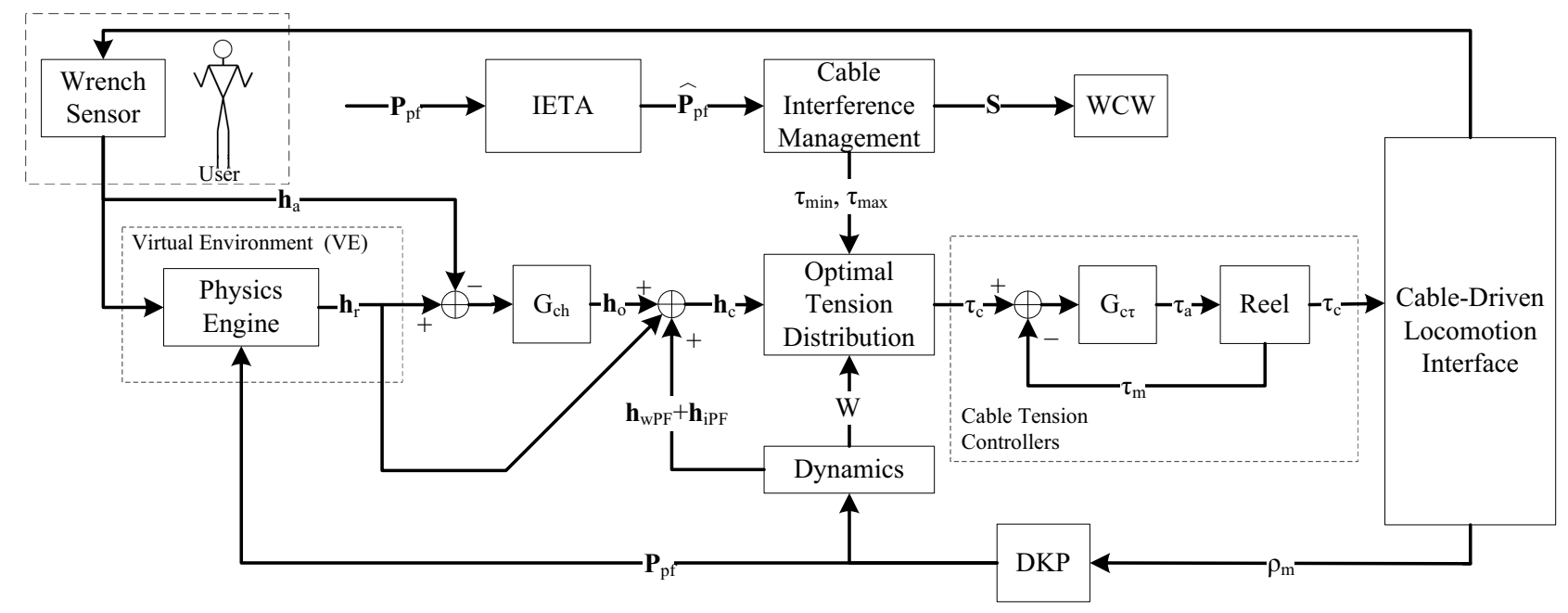

Fig. 1. Control algorithm
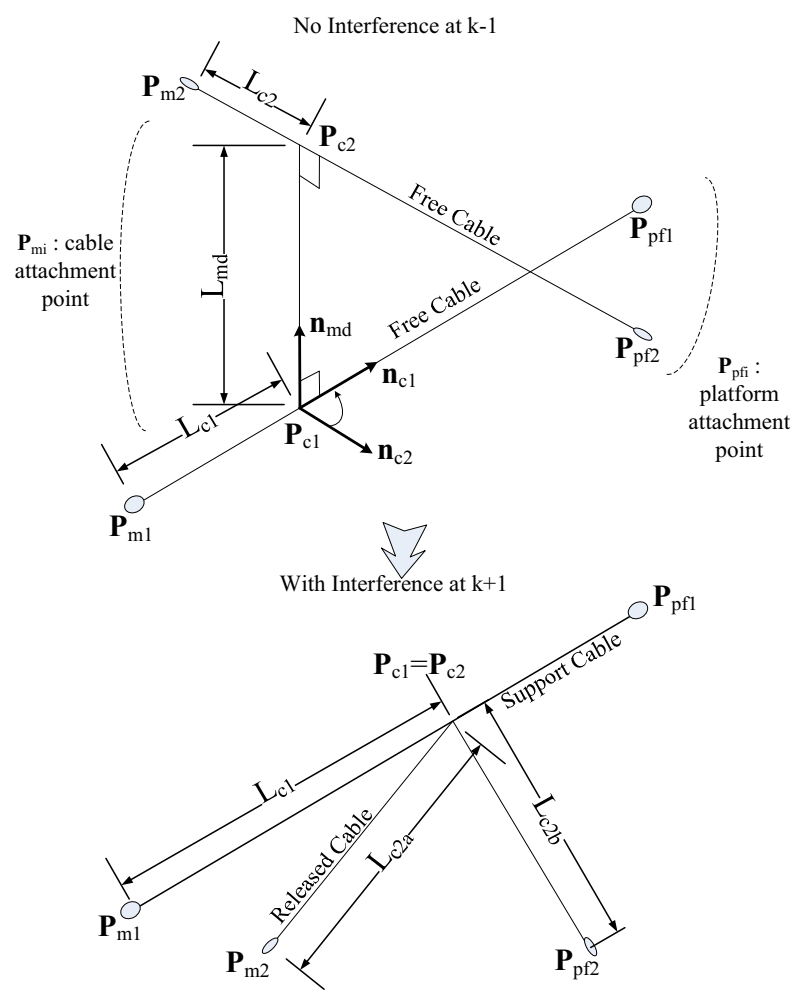

Fig. 2. Interference conditions

active actuation state keeps the platform inside the workspace (step 1). This can result in four distinct cases (step 2):

- any released cable generates a pose inside the workspace (case 1);

- cable 1 released: platform is inside the workspace (case 2);

- cable 2 released: platform is inside the workspace (case 3) and

- any released cable generates a pose outside the work- space (case 4).

When case 1 occurs, the selection of the cable to be released depends on whether the interference involves the cables of a single platform or the cables between both platforms. In some architectures, however, a cable-driven platform may employ more than eight cables, in which case the cable to be released when the tension distribution is homogeneous depends on the total number of released cables. The algorithm therefore attempts to minimize the number of interferences per platform so as to limit their adverse effects: the reduction of the size of the workspace and the increasing potential tension discontinuities. Afterwards, the algorithm selects the cable with the lowest tension as the one to be released.

If cases 2 and 3 occur, the cable to be released is simply the one that allows the platform to remain inside the workspace. The fourth case, where both platforms leave the controllable workspace, is a condition that must never occur, because in this case no cable can be safely released. This corresponds to the pathological Unstable Interference Domain (UID) case that must be treated in the same manner as a watchdog timer, i.e., the whole system is shut down and all reel brakes are activated.

\section{INTERfEREnCE Estimated TIME OF ARrival}

Constraint adjustments are applied before and after an interference occurs in order to achieve a sub-optimal solution given by the OTD algorithm within an allowed relative maximum tension variation between two consecutive computational steps. Indeed, the best solution of the OTD encompasses the following three conditions which may not always be satisfied simultaneously:

- the platform must be in the wrench-closure workspace;

- solution continuity must be satisfied for all cases including interference and

- the solution should be optimized by the OTD. 


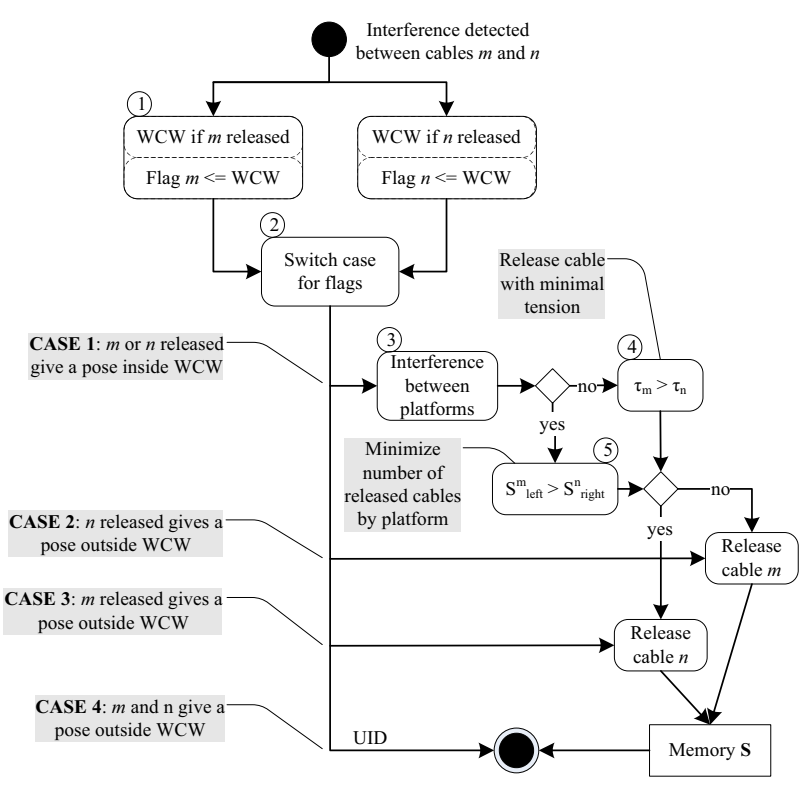

Fig. 3. Cable selection algorithm

It is possible to estimate accurately the time left prior to a collision event if the trajectories of two cables are known. Precisely, in a system for which random trajectories dictate the motion of the platforms, it is only possible to estimate future trajectories by using previously accumulated platform position data, so as to calculate an approximative IETA that afterward can modify OTD constraints in order to reduce or eliminate tension discontinuities. As this IETA quantity is of crucial importance, two approaches for its computation are simulated and their performance compared.

The first method consists of computing the estimated time, $\hat{t}$, using a formulation based on the distance between two cables as defined by (6). The roots of this equation yield an approximation of the time left before an interference event occurs. As it is a quadratic form, its computation is straightforward.

$$
0=\sum_{j=0}^{n} \frac{d^{j} L_{m d}}{d \hat{t}^{j}} \frac{\hat{t}^{j}}{j !}
$$

However, this method can only predict the time left before an interference but fails to provide the point of interference on both cables. In addition, it cannot verify whether this interference point is located in the virtual extension of the cables or not. These issues are addressed by the second method, where the location of an interference point can be computed with (7):

$$
\begin{gathered}
\mathbf{g}_{1} u_{1}+\mathbf{P}_{m 1}=\mathbf{g}_{2} u_{2}+\mathbf{P}_{m 2}, \text { with } \\
0<u_{i}<1 \\
\mathbf{g}_{i}=\sum_{j=0}^{n} \frac{d^{j}\left(\mathbf{P}_{p f i}-\mathbf{P}_{m i}\right)}{d \hat{t}^{j}} \frac{\hat{t}^{j}}{j !} \\
\mathbf{g}_{i}=\left[g_{x}, g_{y}, g_{z}\right]_{i}^{T}
\end{gathered}
$$

The closed-form solution of (7) leads to (11), which corresponds to a quartic equation when $n=2$ and a sextic equation when $n=3$. A valid interference event at estimated time $\hat{t}$ then simply corresponds to a real positive root of (11) within the constraints defined in (8).

$$
\begin{aligned}
0= & \left(g_{z 1} g_{y 2}-g_{y 1} g_{z 2}\right) \Delta P_{m x}+ \\
& \left(g_{x 1} g_{z 2}-g_{z 1} g_{x 2}\right) \Delta P_{m y}+ \\
& \left(g_{y 1} g_{x 2}-g_{x 1} g_{y 2}\right) \Delta P_{m z} . \\
\Delta \mathbf{P}_{m}= & \mathbf{P}_{m 2}-\mathbf{P}_{m 1} \\
\mathbf{P}_{m i}= & {\left[P_{m x}, P_{m y}, P_{m z}\right]_{i}^{T} }
\end{aligned}
$$

The resulting IETA value is expressed in Hertz-second as IETA $=F_{e} \cdot \hat{t}$, where $F_{e}$ is the sampling frequency of the control process. In fact, the IETA simply corresponds to the estimated number of computation steps before an interference event occurs. The algorithm for computing an IETA is called as soon as the distance between two cables drops below a distance threshold $\epsilon_{r}$, and the IETA is only considered when the collision time drops below a given horizon of prediction.

To reduce or eliminate discontinuities, the tension in a cable that is about to be released from an active actuation state must be smooth and must decrease monotonically toward its target minimum tension value, the tension variation rate being determined as a function of the $F_{e} \cdot \hat{t}$ as well as the actual tension $\tau$. Similarly, the tension in the corresponding support cable must be increased or decreased smoothly.

Note that the cable to be released is chosen using the cable selection algorithm presented in section III-B. It is a function of the future workspace, which depends on the estimated platform position $\hat{\mathbf{P}}_{p f i}$ at $\hat{t}$ that is computed using (14). Moreover, this cable serves as a basis with which the smoothness in the transition of tension of all involved cables is subsequently computed.

$$
\hat{\mathbf{P}_{p f i}}=\left.\mathbf{g}_{i}\right|_{\hat{t}}+\mathbf{P}_{m i}
$$

\section{Results for a Normal Gait WALKing EXPERIMENT}

The cable interference management algorithm was applied to the cables of a single platform shown in Fig. 4. On this figure, the cables drawn as dashed lines are oriented downward from the platform while those drawn as solid lines are oriented upward. The estimated weight of each platform, including the 6-DOF wrench sensors, is $15 \mathrm{Kg}$.

\section{A. Simulation parameters}

The chosen trajectory (and its corresponding wrench input) emulates straight normal walking [13] for a walker weighting of about $67 \mathrm{Kg}$, and the input consists of force and torque measurements on a normal floor under the walker's feet that are collected at a sampling frequency of $100 \mathrm{~Hz}$. The trajectory and the wrench applied on the platform are defined for 6-DOF and are presented in [2]. A short video that 


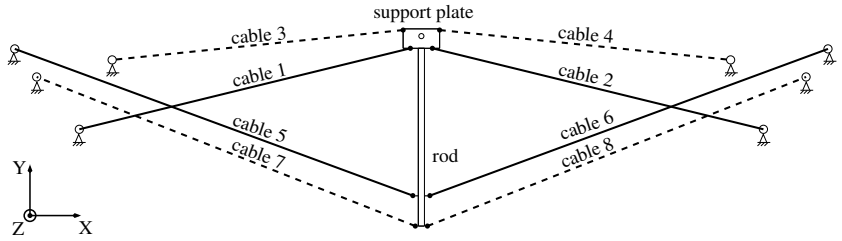

Fig. 4. Top view of a platform (taken from [2])

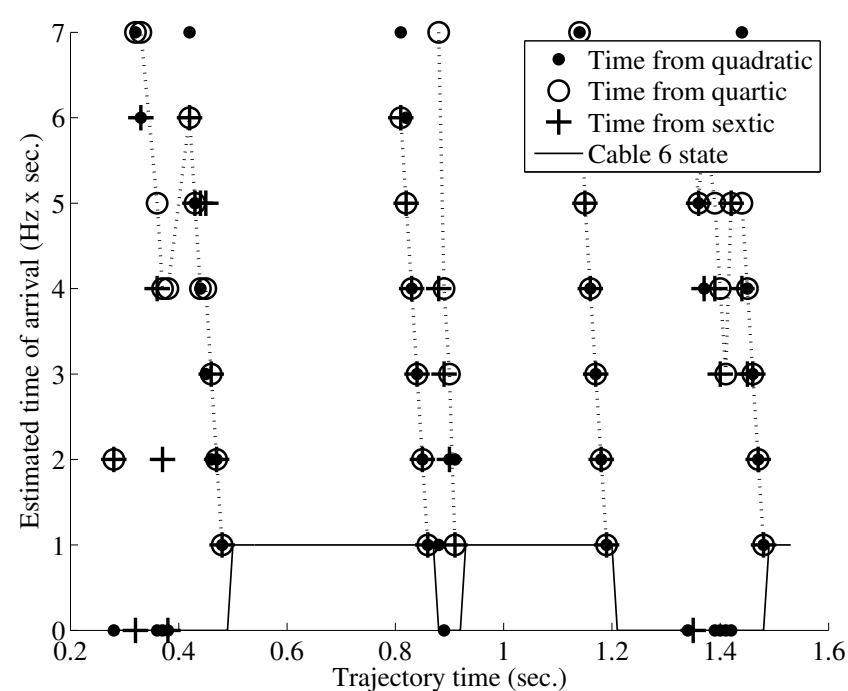

Fig. 5. Interference Estimated Time of Arrival (IETA)

accompanies this paper [14], shows a demonstration of the practical use of the IETA for the $1 / 3$ scale prototype of the Fig. 4. For this demonstration, the chosen trajectory allows the cable number 2 and 6 to interfere each other.

\section{B. IETA analysis}

The IETA analysis is carried out using the trajectory defined previously so as to easily simulate interference events between the cables of one platform. Cable 6 generates an interferences with cable 2 three times over the total cable paths. Fig. 5 shows the beginning and ending of each interference event with its corresponding IETA value at each computation step. This IETA is evaluated using three equations: the quadratic form of the normal distance between the involved cables (equation (6)), the quartic form representing the Euclidean space-time coordinates of each interference, as well as its sextic counterpart (equation (11)).

The simulation are similar for both the quadratic and quartic forms, which is to be expected as the evaluation horizon is short (the best results being obtained with a prediction horizon IET $A<7$ with a sampling rate of 100 $\mathrm{Hz}$ ). However, the performance of the sextic form seems to degenerate even though some data points still match those obtained with the other methods. It is believed that this result may be caused by the numerical errors resulting from the evaluation of the acceleration. Based on the aforementioned results, the quartic formulation is chosen as the preferred method for evaluating interferences and for reducing cable tension discontinuities.
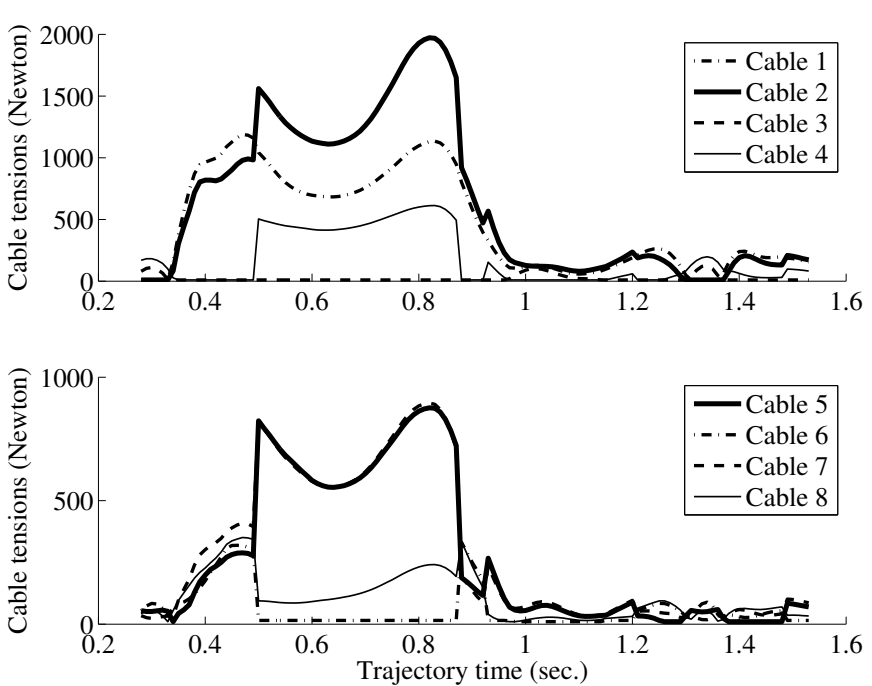

Fig. 6. Cable tension discontinuities without IETA (interference between cables 2 and 6)

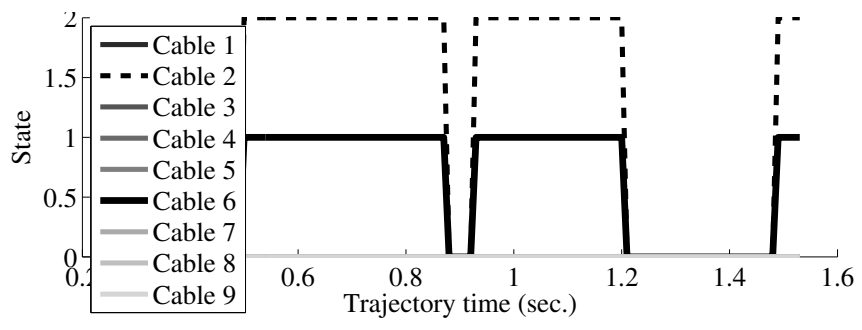

Fig. 7. Cable states $\mathbf{S}$ with an interference between cables 2 and 6

\section{Interference between cables}

The solutions (without IETA evaluation) for cable tensions are plotted in Fig. 6 . When cable 6 is released, the tensions in the other cables exhibit discontinuities that are quite difficult to control, as demonstrated by the tension variation in support cable 2 which is subjected to an increase in tension greater than $700 \mathrm{~N}$ during one computing step. The cable states $\mathrm{S}$ are plotted in Fig. 7 (as seen in section III-A a cable can be only in one of the three states : free, released or support, labelled 0,1 and 2 respectively).

The results of this analysis shows low tensions in some cables. It can be explained by the geometry of the CableDriven Locomotion Interface (CDLI) as the cables whose anchor points are located under the platforms do not need to work against gravity for a trajectory emulating gait, even though they are still needed in order to completely constrain the platform so as to allow any arbitrary trajectory as well as any force feedback generated by the virtual environment such those involved in the action of kicking a ball with the foot.

As mentioned earlier, the IETA defined in section IV is evaluated so as to reduce discontinuities and the results are plotted in Fig. 8. Cable tension control is always used whenever some cables are in interference. For removing discontinuities, adjustment of constraints in the Optimal Tension Distribution (OTD) algorithm in function of the $F_{e} \cdot \hat{t}$ 

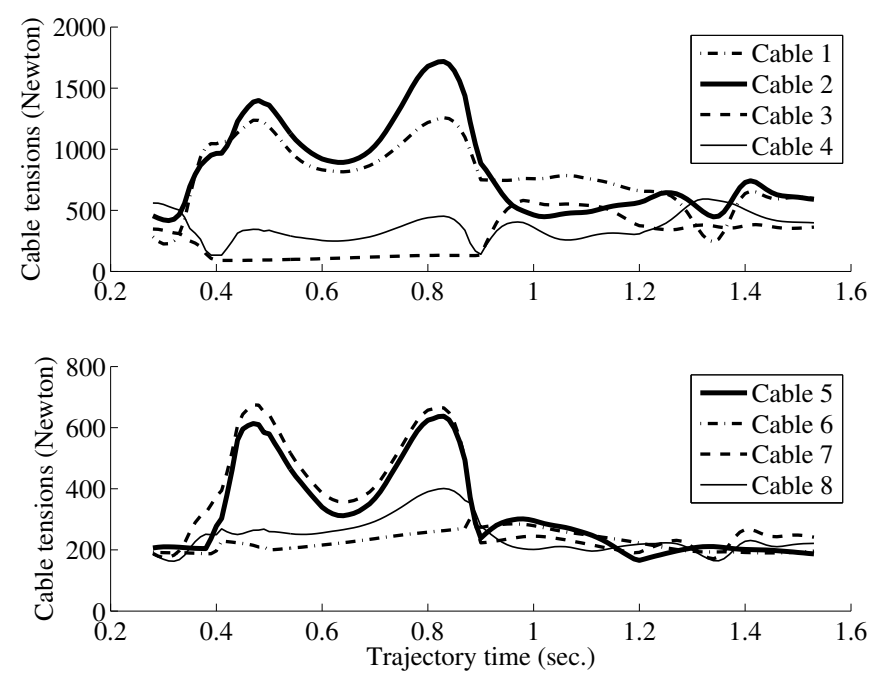

Fig. 8. Correction of cable tensions with IETA algorithm

have to be performed for each prediction of an interference. Also, the minimal tensions is evaluated for reducing cable sagging.

Finally, the video in [14] shows that it is possible to pull the released cable number 6 without moving the virtual foot liked to the robotic platform.

\section{CONCLUSIONS AND Future WORKS}

\section{A. Conclusion}

The main concern when managing interferences between cables in a cable-driven mechanism is to ensure that the solutions (with respect to the cable tensions) are continuous at all times in order to avoid instabilities. Unfortunately, this cannot be achieved using standard quadratic programming or Optimal Tension Distribution (OTD) algorithm. As a matter of fact, when a cable is released from an active actuation state, its tension can drop from a large value to a minimum tension in only one computation step, thereby suddenly submitting the other cables to high tension values which, as a result, can generate mechanical vibrations and instabilities.

This paper describes a predictive algorithm that combines an IETA parameter with a hyperplane intersection calculation procedure to reduce cable tension discontinuities while using an optimal tension distribution algorithm. This procedure can be theoretically improved in accuracy by increasing the order of the Taylor series that approximates each cable trajectory.

\section{B. Future Works}

Cable interference is characterized by friction forces at the interference location, the deflection of the support cable required for static and dynamic equilibrium, cable sagging, cable elongation, and the precision of both the mechanical and data acquisition systems. Each of these factors generates errors during wrench computations which translate into platform movement uncertainties as the cable tensions cannot exactly balance the wrench applied on each platform. Future work will address the evaluation of cable sagging.
Performance criteria on cable tensions will be defined to ensure that the effect of gravity on the cables can be neglected.

\section{ACKNOWLEDGMENTS}

The authors would like to acknowledge the financial support of the Natural Sciences and Engineering Research Council (NSERC) through its strategic program. The engineering work on the data acquisition system and the computer drivers by Denis Ouellet and Sylvain Comptois from the LVSN team (Laboratoire de Vision et des Systèmes Numériques) at Laval University is also acknowledged.

\section{REFERENCES}

[1] M. J.-D. Otis, M. Mokhtari, C. Du Tremblay, D. Laurendeau, F.-M. De Rainville, and C. M. Gosselin, "Hybrid control with multi-contact interactions for 6dof haptic foot platform on a cable-driven locomotion interface," in Symposium on Haptics Interfaces for Virtual Environment and Teleoperator Systems 2008 - Proceedings, Haptics, Piscataway, NJ 08855-1331, United States, 2008, pp. $161-168$.

[2] S. Perreault and C. M. Gosselin, "Cable-driven parallel mechanisms: Application to a locomotion interface," Journal of Mechanical Design, Transactions of the ASME, vol. 130, no. 10, p. 10230, 2008.

[3] K. Maeda, S. Tadokoro, T. Takamori, M. Hiller, and R. Verhoeven, "On design of a redundant wire-driven parallel robot warp manipulator," in Proceedings of the IEEE International Conference on Robotics and Automation, vol. 2, Detroit, MI, USA, 1999, pp. 895 - 900.

[4] J.-P. Merlet, "Analysis of the influence of wires interference on the workspace of wire robots," Advances in Robot Kinematics, pp. 211218, June 2004

[5] S. Fang, D. Franitza, M. Torlo, F. Bekes, and M. Hiller, "Motion control of a tendon-based parallel manipulator using optimal tension distribution," IEEE/ASME Transactions on Mechatronics, vol. 9, no. 3, pp. $561-568$, September 2004.

[6] M. Gouttefarde, J.-P. Merlet, and D. Daney, "Wrench-feasible workspace of parallel cable-driven mechanisms," in Proceeding of the IEEE International Conference on Robotics and Automation, Roma, Italy, april 2007, pp. 1492 - 1497.

[7] K. Kozak, Q. Zhou, and J. Wang, "Static analysis of cable-driven manipulators with non-negligible cable mass," IEEE Transactions on Robotics, vol. 22, no. 3, pp. 425 - 33, 062006.

[8] P. Zhuang and Z. Yao, "Dynamics and control of cable-suspended parallel robots for giant telescopes," in Proceedings of SPIE - The International Society for Optical Engineering, vol. 6267 II, Orlando, FL, United States, 2006, pp. 62673 -.

[9] Y. Zheng and X. Liu, "Optimal tension distribution of wire-driven parallel manipulators," Jixie Gongcheng Xuebao/Chinese Journal of Mechanical Engineering, vol. 41, no. 9, pp. 140 - 145, 2005.

[10] D. Theodorakatos, E. Stump, and V. Kumar, "Kinematics and pose estimation for cable actuated parallel manipulators," in 2007 Proceedings of the ASME International Design Engineering Technical Conferences and Computers and Information in Engineering Conference, DETC2007, vol. 8 PART B, Las Vegas, NV, United States, 2008, pp. $1053-1062$.

[11] A. Pott, D. Franitza, and M. Hiller, "Orientation workspace verification for parallel kinematic machines with constant leg length," in Proceedings of the Mechatronics and Robotics, Aachen, Deutschland, 2004.

[12] Y. Wischnitzer, N. Shvalb, and M. Shoham, "Wire-driven parallel robot: Permitting collisions between wires," International Journal of Robotics Research, vol. 27, no. 9, pp. 1007 - 1026, 2008.

[13] B. J. McFadyen and F. Prince, "Avoidance and accomodation of surface height changes by healty, community-dwelling, young, and elderly men," Journal of Gerontology: Biological sciences, vol. 57A, no. 4, pp. B166-B174, 2002.

[14] M. J.-D. Otis, "Interference eta on a 6-dof cable-driven haptic platform," accompany video, 2008. 